\title{
Distance Vector Routing for Public Transportation Vehicular Networks: Performance Evaluation on a Real Topology
}

\author{
Sabrina Gaito, Dario Maggiorini, Elena Pagani, Gian Paolo Rossi \\ Università degli Studi di Milano \\ Via Comelico 39, 20135 Milano, Italy \\ Email: firstname.lastname@unimi.it
}

\begin{abstract}
In this last two years an increasing number of scientists has devoted his attention to Vehicular Ad hoc NETworks (VANETs) as a special case of the more generic Mobile Ad hoc NETworks (MANETs). A public transportation network used as a VANET backbone may be a viable solution - under certain circumstances - also thanks to its almost-complete area coverage and the pre-scheduled repetitive paths. The aim of this paper is to compare performances of distance vector (DV) routing strategies when applied to a VANET moving as for a real city topology. Our two main contributions are: firstly, to compare results with similar - more canonical - experiments using a grid, and secondly, to outline differences between various DV metrics.

By means of simulations we demonstrate that, in the considered network scenario, a distance vector-based routing algorithm can perform well and more complex - and resources hungry protocols are no longer strictly needed. Moreover, simulations based on a grid topology reproduce the behavior of a real city in a limited way.
\end{abstract}

Index Terms-Ad hoc networks, Vehicular networks, Routing protocols, Distance Vector, Delay Tolerant Networks.

\section{INTRODUCTION}

In the last few years, thanks to the increasing diffusion of broadband wireless technology, we have seen the dawn of infrastructures for large-scale mobile communication and service provisioning. Among these infrastructures, the ones using inter-vehicular communication witnessed to be an attractive environment for ad-hoc applications due to vehicles density and limited speed in urban areas. Vehicular Ad hoc NETworks (VANETs) can be considered as a specific case of Mobile Ad hoc NETworks (MANETs) where a number of constraints are applied to the nodes; in particular: $(i)$ movement is constrained by the urban plan, (ii) speed is bounded by the law, (iii) queuing happens only at pre-determined points, and $(i v)$ power management is no longer a critical issue.

Routing strategies in VANET environments adopt a storecarry-and forward strategy typical of Delay Tolerant Networks (DTNs) [1]: a message may require a considerable amount of time to traverse the network while sitting in the buffer of a carrier node waiting to encounter a valid forwarder. For the above reasons, routing algorithms are required to minimize end-toend delay, resources usage, and loss probability. Routing in

This work is supported by the Italian Ministry of University and Research, project CARTOON, Contract No. 2006091851
VANETs is commonly performed by means of a positionalrouting strategy [2] combined with a location service [3] to acquire the target position. Using this approach, delivery ratio is the only parameter taken into account for optimization, while transmission delay and network usage are left for a besteffort policy. On the one hand, geo-location requires to equip all vehicles with a Global Positioning System (GPS) receiver and the routing protocol needs to take into account (sometimes unreliable) destination coordinates. On the other hand, location services may use packet replication (with consequent high resources usage) and scalability is not always guaranteed [4] [5] [6] [7].

Among VANETs, public transportation systems represent an interesting case study due to their regular movements both on a pre-assigned path and over time. This regularity can be exploited to relax requirements from routing protocols while increasing their performances. In particular, a VANET network backbone may be of interest in areas where cellular networks are not installed like rural areas or underdeveloped countries, and situations where sensor networks are too expensive to deploy or do not scale well like fine-grained pollution monitoring in a big city.

This paper is the evolution of a previous work [8] where performances of distance vector (DV) routing protocols have been compared using a standard grid topology. The previous contribution aimed to identify the best strategy to achieve a satisfactory delivery while minimizing resources usage. Results indicated that DV-based routing performance may be satisfactory for a urban DTN and, while metrics based only on inter-vehicle contacts frequency are prone to count to infinity problems, hybrid metrics considering both hops count and contacts opportunities proved to have a better performance than standard distance vector, which is based solely on hops count. In this paper, improved experiments are performed using the actual topology of the city of Milano: a huge number of nodes are simulated moving as for the real public transportation system timetable. The goal here is mainly to understand if the proposed DV metrics are actually a good choice also for a more realistic environment. Moreover, comparison of results will lead to some considerations about the approximation degree introduced by using a grid topology rather than a real, and more complex, one. 


\section{RELATED WORK}

In the last years some efforts have been devoted by the scientific community to optimize and adapt existing routing strategies to the specific environment we are considering here, which is, as previously said, an hybrid network between a DTN and a VANET. As a result, an increasing literature can be found on delay-aware routing protocols and routing for vehicular networks. In this section we will present the ones more closely related to the scenario considered here.

In [9] connectivity between nodes on a broad area is provided by means of special roaming nodes, namely message ferries, conveying packets and using different communication schemes, involving nodes mobility, to receive and forward messages.

VANET routing protocols are usually designed for urban environments where vehicles are equipped with wireless connectivity and a GPS device. These protocols relies on geographic forwarding [2] for packet routing: the next hop is the vehicle in radio range which is closest to the destination and identified by a location service [3]. In the Spatially Aware packet Routing (SAR) algorithm [10], nodes are aware of the street map and compute a set of Anchor Points (APs) along streets to help the routing process. Forwarding from one AP to the next is performed using Geographic Forwarding. Spatial and TrafficAware Routing (STAR) [11] enhances SAR, allowing vehicles to collect and spread traffic information used to weight streets on the road map: the higher the vehicular traffic, the higher the score applied on a certain street. In Anchor-based Street and Traffic Aware Routing (A-STAR) [12], road map streets are weighted on the basis of known bus routes.

BUSNet [13] is, at the best of our knowledge, the first and more prominent work proposing a bus backbone in a urban environment to allow inter-vehicle routing. Unluckily, delay tolerant communication has not been taken into account by authors; the paper suggests to rely on the aid of other vehicles (e.g., cars) to avoid partitions in the backbone and to obtain point-to-point communication.

Nevertheless, delay tolerant networks based only on buses are winning an increasing attention in these last few years.

Rural environments have been studied in papers like [14] and [15]. In the first a link state routing policy is proposed thanks to the determinism of the routes, while in the second, the message ferrying approach has been successfully applied to the rural India.

Urban environments are more challenging due to the complexity of their topology and hard encounter predictability. In [16] the contacts features of a campus bus transportation system are addressed, while in [17] a wider scenario has been considered: the Shanghai public transportation system. In particular, in the second paper a routing scheme based on a contact oracle exploiting the total contact duration between every two lines has been compared with epidemic and earliest delivery routings.

\section{BEYOND MANHATTAN}

The Public Transportation System (PTS) of Milano is a complex system extending above and below ground and spanning more than 70 lines inside the city. Coverage for these lines be seen in Fig. 1. As can be observed from the map, the

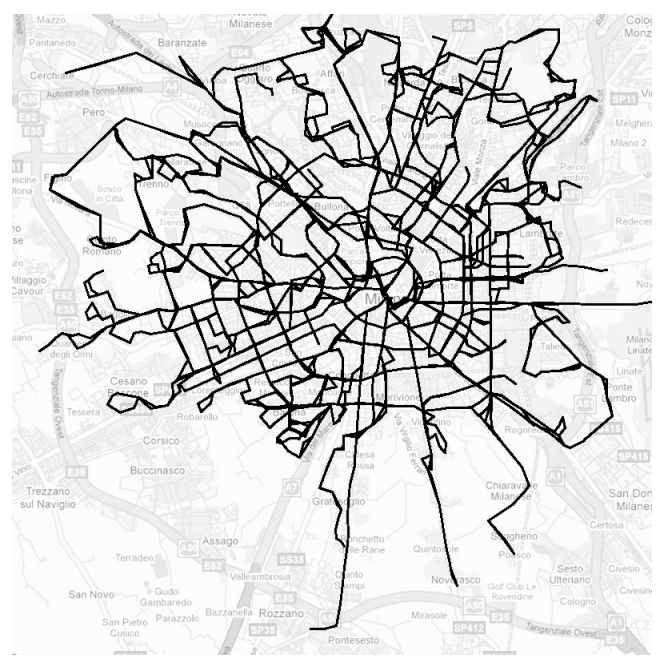

Fig. 1: Ground transportation lines used for simulation.

overall structure is clearly not grid-like: crosses between bus lines may occur at any time and there is no constant space between intersections.

In this paper we will be using a model based on the above topology in order to simulate the behavior of a MANET.

The movement model has been obtained using information available from the public transportation company website [18] and the google maps service. The address of every bus stop has been used to derive its GPS coordinates which have later been converted to Cartesian coordinates. Nodes along each line move between coordinates accordingly to real timetables. Taking into account departure times from line heads and keeping the correct passage rate on intermediate bus-stops we estimated a speed between 2.4 and $4.8 \mathrm{~m} / \mathrm{s}$. Buses leave the line head at the scheduled time as for the real system; upon completing a full round they will queue, if needed, at the first bus-stop behind the coach waiting for the next scheduled start. If there are already two buses in line, then we consider the line to be overpopulated and the bus goes out of service. The decision to send to recovery a bus when two others are on queue at the first bus-stop have been taken based on practical observation: we rarely see more than one bus ready to leave the line head. Simulated bus population seems to be in line with the observer real world behavior and information made available from the public transportation company.

\section{Application Scenario}

The application scenario we are going to target is related to lazy low-cost information services in urban environment. This kind of context has already been addressed in car-to-car environment, like in [19]; in our case we are considering free 
push services for the citizens, like e-mail on the go or a twitterlike microblogging. During operations the traffic is supposed to be generated from the users of the PTS - from inside the coaches or along the streets - and directed to some location which we can assume is covered by at least by one bus line. As for the way traffic is generated, routing takes place between a bus of a certain line and another line, and not between nodes: if the next hop to a destination is the line $l$, any bus belonging to line $l$ will be a valid forwarder.

In order to create a distribute routing system each node advertises its distance to every other known destination using beacons. Nearby nodes computes their own distance when forwarding through the advertiser based on the local adopted metric. An advertising node represents the line it belongs to; therefore, every encountered node is used to estimate the distance to its line. When several buses belonging to the same line are met the most convenient distance is taken for that line. A known line is selected as next hop to a certain destination based on the best distance value. Each node maintains only the best path to each known destination, that is the one with the most favorable distance.

\section{Distance Vector Metrics}

In our performance evaluation we consider distance vector algorithms based on four different metrics:

1) Hop Count Metric (HCM) - to minimize the number of traversed nodes

2) Mean Metric (MM) - to maximize delivery chances while reducing buffer usage

3) Fair Metric (FM) - to maximize delivery chances

4) Worst Case Metric (WCM) - to minimize the chances of an undelivered message

HCM is the original metric used in the distance vector algorithm for infrastructure-based networks. Distance is calculated as the number of forwards required to reach the destination; a lower distance value implies a better path.

The other metrics take into account the contact time defined as the sum of the contact durations over a candidate path. The motivation behind this is that path selection in a DTN relies on node meetings, and longer contact times may imply higher opportunities for data exchange. Each node keeps track of the time it has been in contact with any other node (Contact Time or $C T$ ) and calculates the distance as $\frac{C T}{E T}$, where the $E T$ is the elapsed time (i.e., the time for which the bus has been on duty). Thus the distance between a node $A$ and an encountered node $B$ is:

$$
d(A, B)=\frac{C T_{A, B}}{E T_{A}}
$$

Please note, this is not a distance in a geometric way since $d(A, B)$ may differ from $d(B, A)$, allowing for asymmetric round-trip paths.

In the following we will indicate the source, destination, and relay candidate as $S, D$, and $R$ respectively.

In MM the distance from $S$ to $D$ when routing through $R$ is estimated by $S$ as the distance advertised by $R$ conveniently mediated by its distance to $R$, as shown below.

$$
d(S, D)=\frac{1}{2} d(R, D)+\frac{1}{2} d(S, R)
$$

Distance computation starts from the $D$, which advertises a distance 1 from itself. The left-hand side of the sum, whose value at first hop will be $d(D, D)$ (or 1 ), will be predominant and halved at each step. The contribution of the second factor will help MM to choose the best compromise between global delivery chances and quickest encounter opportunity with the first hop. With this kind of approach buffer usage will be reduced since the earlier the first hop is encountered the less time a packet will sit in the local buffer. With this metric the distance of a path made of $n-1$ hops and whose forwarding nodes are labelled from $N_{1}(S)$ to $N_{n}(D)$, is:

$$
d\left(N_{1}, N_{n}\right)=\left(\sum_{i=1}^{n-2} \frac{1}{2^{i}} d\left(N_{i}, N_{i+1}\right)\right)+\frac{1}{2^{n-2}} d\left(N_{n-1}, N_{n}\right)
$$

In $\mathrm{MM}$ an higher distance implies a better path.

In FM $S$ estimates the distance to $D$ using the same considerations as MM but the product between the distance with $R$ and its advertised distance to $D$ is computed.

$$
d(S, D)=d(S, R) \times d(R, D)
$$

In this case the hop count is considered implicitly. Each node added to the path multiplies the distance by a factor usually much lower than 1; in FM, as in MM, a higher distance implies a better path. With this metric the overall distance of a path made of $n-1$ hops is:

$$
d\left(N_{1}, N_{n}\right)=\prod_{i=1}^{n-1} d\left(N_{i}, N_{i+1}\right)
$$

WCM, as suggested by the name, considers the worse distance on the whole path to estimate the goodness of a route to the destination. $S$ compares the distance advertised by $R$ with the observed distance and takes the lower value. Among all possible next hops the one with higher distance value is selected: forwarding will thus take place along the less worst path, minimizing the chances to leave a packet undelivered.

\section{Vi. Performance Evaluation}

\section{A. Experiments Setting}

In the experiments we assume the use of Wi-Fi technology, therefore a radio range of 100 meters has been considered.

All simulations cover a full cycle of the public transportation service: from the time when the first scheduled bus leaves (around 5 A.M.) up to the end of the last scheduled bus. In order to isolate a full cycle we reported all scheduled departures between midnight and 5 A.M. as if happening in the previous day, this is why experiments last more than 24 hours.

In order to adhere to the application scenario each bus will generate a message every 5 minutes and the destination will be set to another randomly chosen bus line. When the message is generated it is placed in the local memory store until a 
forwarding opportunity arises. When an encounter happens all the messages in the buffer are checked and forwarded if the in-range bus belongs to a line which is a valid next hop. When a bus reaches the end of the line it may or may not put itself on queue and wait for another scheduled departure. If the bus stays on the queue it will hold all its data and keep generating messages while waiting. If, in the other case, the bus will go out of service all content will be pushed to the first bus waiting in line. In the case there are no bus available - because there are no more scheduled departures - all the stored messages are dropped.

Policies based on contacts frequency are prone to the count to infinity issue because nodes may propagate an outdated routing information base and create loops. In order to prevent this occurrence, we adopted a split horizon technique: the next hop is advertised together with the distance value to the destination, in this way the next hop will not consider the advertising node has a possible forwarder to the destination. Nevertheless, we need anyway to detect when a packet get caught in a loop. Every message brings along its list of visited nodes; as soon as a packet is forwarded for the second time to the same node it is considered to be in a loop and will be immediately dropped. Mind, a packet may use the same line for two times but will not get discarded as long as it is not hopping on the same bus twice.

\section{B. Simulation Results}

In this section we will summarize the most significative results of the simulations described in the previous section.

In Fig. 2 traffic delivery profiles are shown for the various metrics when node speed is $2.4 \mathrm{~m} / \mathrm{s}$. As it can be observed,

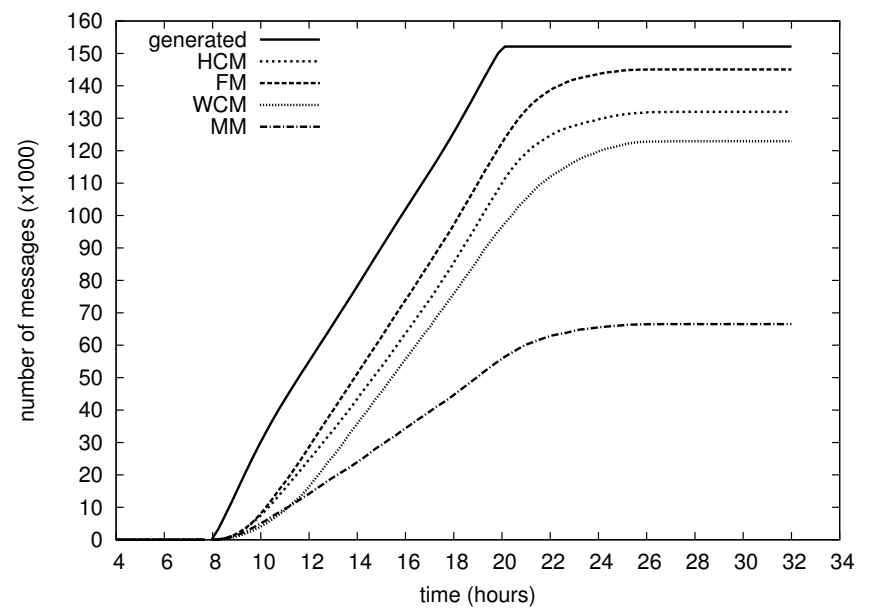

Fig. 2: Packet delivery over time with node speed of $2.4 \mathrm{~m} / \mathrm{s}$.

performances are quite acceptable, but for the MM metric, and results from previous experiments on the grid layout ( [8]) are almost confirmed. FM behaves very well in term of delivered packets, followed by HCM and WCM while MM delivery ratio is below $50 \%$ of the generated traffic. Differently from the previous paper, the performances attained by FM is visibly greater than HCM. This is an indication that HCM may not really be the best choice on a complex topology. Histograms of number of hops and end-to-end delay for FM are reported in Fig. 3 and 4 respectively.

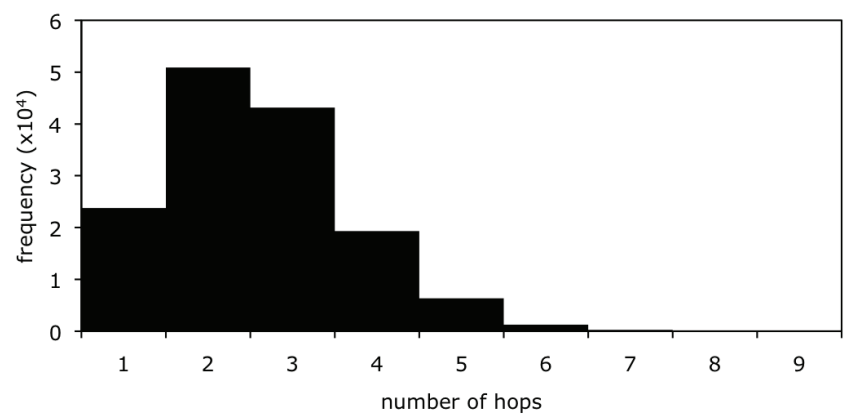

Fig. 3: Hop count histogram for FM with node speed of 2.4 $\mathrm{m} / \mathrm{s}$. Mean is 2.6, median is 2.0, standard deviation is 1.1 .

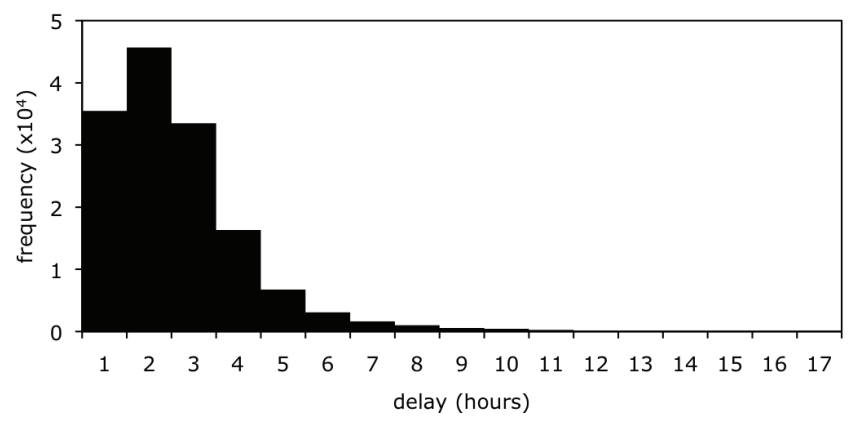

Fig. 4: End-to-end delay histgram for FM with node speed of $2.4 \mathrm{~m} / \mathrm{s}$. Mean is 2.1, median is 1.8 , standard deviation is 1.6.

Regarding the MM metric, the undelivered packets are reported in the $\operatorname{logs}$ as discarded due to count to infinity. If we have a look to Tab. I, we can see that, despite the very high drop ratio, delivered packets offer performances in term of end-to-end delay and hop counts of the same order of magnitude of the other metrics. Thus, the only real problem form MM at this speed is the presence of loops in the forwarding paths.

\begin{tabular}{c|c|c|c|c|}
\cline { 2 - 5 } & $\begin{array}{c}\text { Speed } \\
(\mathrm{m} / \mathrm{s})\end{array}$ & $\begin{array}{c}\text { Average } \\
\text { delay } \\
\text { (hours) }\end{array}$ & $\begin{array}{c}\text { Average } \\
\text { hop } \\
\text { count }\end{array}$ & $\begin{array}{c}\text { Delivery } \\
\text { ratio } \\
(\%)\end{array}$ \\
\hline \hline \multirow{3}{*}{ HCM } & 2.4 & 2.6 & 2.8 & 86 \\
\cline { 2 - 5 } & 3.6 & 2.4 & 1.9 & 86 \\
\cline { 2 - 5 } & 4.8 & 2.3 & 1.9 & 84 \\
\hline \hline \multirow{3}{*}{ FM } & 2.4 & 2.1 & 2.6 & 95 \\
\cline { 2 - 5 } & 3.6 & 1.9 & 2.4 & 93 \\
\cline { 2 - 5 } & 4.8 & 1.8 & 2.3 & 92 \\
\hline \hline \multirow{3}{*}{ WCM } & 2.4 & 2.9 & 5.3 & 80 \\
\cline { 2 - 5 } & 3.6 & 2.5 & 5.5 & 84 \\
\hline \hline \multirow{3}{*}{ MM } & 4.8 & 2.1 & 5.6 & 86 \\
\cline { 2 - 5 } & 2.4 & 2.2 & 2.9 & 43 \\
\cline { 2 - 5 } & 3.6 & 1.7 & 2.1 & 48 \\
\hline
\end{tabular}

TABLE I: Delivery statistics. 
Moreover, experiments on the grid topology exposed a periodic behavior in packet forwarding with a stair-shaped profile. With this complex topology we no longer experience this behavior maybe thanks to the huge number of nodes.

By increasing the speed of nodes to $4.8 \mathrm{~m} / \mathrm{s}$ we obtain the delivery profiles depicted in Fig. 5. Form this figure we

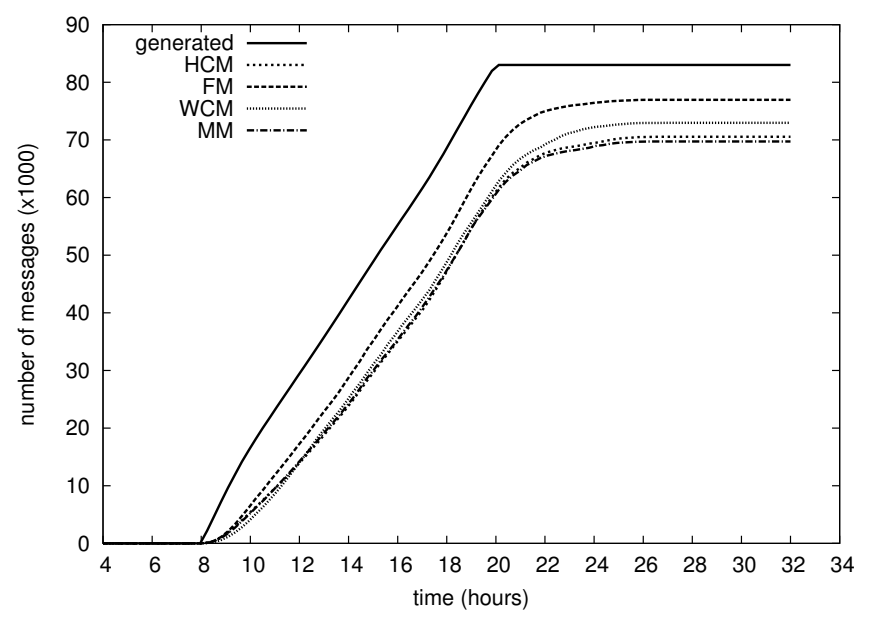

Fig. 5: Packet delivery over time with node speed of $4.8 \mathrm{~m} / \mathrm{s}$.

notice that metrics order by performance is unchanged and the delivery ration (reported in Tab. I) is also almost unchanged but, once again, for MM. MM reports a tremendous increase in delivery ratio; a possible explanation is that increasing the speed reduces the contact opportunity and push the left-hand side of the MM distance formula toward 0; the right-hand side will keep halving each hop, making MM to behave in a way very similar to HCM. Coming back to Tab. I, we can also observe that for every metric average delivery time decreases when speed increases, confirming results from the grid experiments.

Moreover, also from Tab. I, we can see that the number of hops is very low in all cases, thanks to the optimized transportation planning, and average delivery time are always acceptable for a urban environment (a bit more than 2 hours).

As a final remark, from all the experiments we came to the understanding that the best metric to apply in this specific environment is no long the HCM but it is FM. FM exposes the best delivery ration and the best latency for all speeds at the cost of slightly increasing the number of hops when compared to $\mathrm{HCM}$.

\section{CONCLUSION AND FUtURE WORK}

In this paper we investigated various issues about VANETs routing protocols applied to public transportation systems. In particular, we discussed the actual reliability of simulation results when using a grid topology compared to a real one. Some performance indexes, like end-to-end delay, proved to follow the same trend; on the contrary delivery ratio seems to be sensibly lower and hop-count based metrics do not seem to be any longer the best solution. In our experiments, a distance metric based on encounter opportunities outperforms the hopcount metric both in term of delivery ratio and end-to-end delay. The obtained results hints to explore routing algorithms based on encounter probability for public transportation system in urban environment.

In the future we are planning to devise a routing strategy based on encounter probability and to validate the presented results on other public transportation topologies and with real traffic data.

\section{REFERENCES}

[1] S. Jain, K. R. Fall, and R. K. Patra, "Routing in a Delay Tolerant Network," in Proceedings of ACM SIGCOMM'04, 2004, pp. 145-158.

[2] S. Giordano and I. Stojmenovic, Position based routing algorithms for ad hoc networks: a taxonomy. NJ:Springer, 2004, pp. 103-136.

[3] R. Friedman and G. Kliot, " Location Services in Wireless Ad Hoc and Hybrid Networks: A Survey," Department of Computer Science, Technion, Tech. Rep. CS-2006-10, 2006.

[4] J. Li, J. Jannotti, D. D. Couto, D. Karger, and R. Morris, "A scalable location service for geographic ad-hoc routing," in Proceedings of ACM MOBICOM'O0, 2000, pp. 120-130.

[5] S.-C. M. Woo and S. Singh, "Scalable Routing Protocol for Ad Hoc Networks," Wireless Networks, vol. 7, no. 5, pp. 513-529, 2001.

[6] C. T. Cheng, H. L. Lemberg, S. J. Philip, E. van den Berg, and T. Zhang, "SLALoM: A scalable location management scheme for large mobile ad-hoc networks," in Proceedings of IEEE WCNC'02, 2002.

[7] C. E. Palazzi, S. Ferretti, M. Roccetti, and M. Gerla, "How do you quickly choreograph inter-vehicular communications? a fast vehicle-tovehicle multi-hop broadcast algorithm, explained," in Proc. of the $3^{\text {rd }}$ IEEE Consumer Communications and Networking Conference, 2007.

[8] F. Giudici, D. Maggiorini, and G. Rossi, "Performance Evaluation of Distance Vector-based Routing Protocols for Public Transportation Vehicular Networks," in Proceedings of $1^{\text {st }}$ IFIP Wireless Days Conference, 2008.

[9] W. Zhao, M. Ammar, and E. Zegura, "A message ferrying approach for data delivery in sparse mobile ad hoc networks," in MobiHoc '04: Proceedings of the 5th ACM international symposium on Mobile ad hoc networking and computing. New York, NY, USA: ACM, 2004, pp. 187-198.

[10] J. Tian, L. Han, and K. Rothermel, "Spatially aware packet routing for mobile ad hoc inter-vehicle radio networks," Intelligent Transportation Systems, 2003. Proceedings. 2003 IEEE, vol. 2, pp. 1546-1551 vol.2, Oct. 2003.

[11] F. Giudici and E. Pagani, "Spatial and traffic-aware routing (star) for vehicular systems," High Performance Computing and Communications, 2005. Proceedings. Springer, vol. 3726, pp. 77-86, 2005.

[12] B.-C. Seet, G. Liu, B.-S. Lee, C.-H. Foh, K.-J. Wong, and K.-K. Lee, A-STAR: A Mobile Ad Hoc Routing Strategy for Metropolis Vehicular Communications, 2004. [Online]. Available: http://www.springerlink.com/content/hjurea7cew8gvnrp

[13] K. juan Wong, B. sung Lee, B. chong Seet, G. Liu, and L. Zhu, "Busnet: Model and usage of regular traffic patterns in mobile ad hoc networks for inter-vehicular communications," in Proc. ICT 2003, 2003.

[14] M. Demmer and K. Fall, "Dtlsr: delay tolerant routing for developing regions," in NSDR '07: Proceedings of the 2007 workshop on Networked systems for developing regions. New York, NY, USA: ACM, 2007.

[15] A. Pentland, R. Fletcher, and A. Hasson, "Daknet: rethinking connectivity in developing nations," Computer, vol. 37, no. 1, pp. 78-83, Jan. 2004.

[16] X. Zhang, J. Kurose, B. N. Levine, D. Towsley, and H. Zhang, "Study of a bus-based disruption-tolerant network: mobility modeling and impact on routing," in Proceedings of the $13^{\text {th }}$ annual ACM international conference on Mobile computing and networking. New York, NY, USA: ACM, 2007, pp. 195-206.

[17] M. Sede, X. Li, D. Li, M.-Y. Wu, M. Li, and W. Shu, "Routing in largescale buses ad hoc networks," in Proc. IEEE Wireless Communications and Networking Conference, 2008, pp. 2711-2716.

[18] "Azienda trasporti milanesi," http://www.atm-mi.it/.

[19] C. Palazzi, M. Roccetti, and S. Ferretti, "An inter-vehicular communication architecture for safety and entertainment," IEEE Transactions on Intelligent Transportation Systems, vol. 10, no. 3, Sep 2009. 